\title{
Enabling Lorentz boosted frame particle-in-cell simulations of laser wakefield acceleration in quasi-3D geometry
}

\author{
Peicheng $\mathrm{Yu}^{\mathrm{a}}$, Xinlu $\mathrm{Xu} \mathrm{u}^{\mathrm{a}, \mathrm{b}}$, Asher Davidson ${ }^{\mathrm{b}}$, Adam Tableman ${ }^{\mathrm{b}}$, Thamine \\ Dalichaouch $^{\mathrm{b}}$, Fei Li ${ }^{\mathrm{c}}$, Michael D. Meyers ${ }^{\mathrm{b}}$, Weiming An ${ }^{\mathrm{a}, \mathrm{b}}$, Frank S. \\ Tsung $^{\mathrm{b}}$, Viktor K. Decyk ${ }^{\mathrm{b}}$, Frederico Fiuza ${ }^{\mathrm{d}}$, Jorge Vieira ${ }^{\mathrm{e}}$, Ricardo A. \\ Fonseca $^{\mathrm{e}, \mathrm{f}}$, Wei Lu ${ }^{\mathrm{c}}$, Luis O. Silva ${ }^{\mathrm{e}}$, Warren B. Mori ${ }^{\mathrm{a}, \mathrm{b}}$ \\ ${ }^{a}$ Department of Electrical Engineering, University of California Los Angeles, Los \\ Angeles, CA 90095, USA \\ ${ }^{b}$ Department of Physics and Astronomy, University of California Los Angeles, Los \\ Angeles, CA 90095, USA \\ ${ }^{c}$ Department of Engineering Physics, Tsinghua University, Beijing 100084, China \\ ${ }^{d}$ SLAC National Accelerator Laboratory, Menlo Park, CA 94025, USA \\ ${ }^{e}$ GOLP/Instituto de Plasma e Fusão Nuclear, Instituto Superior Técnico, Universidade \\ de Lisboa, 1049-001, Lisbon, Portugal \\ ${ }^{f}$ ISCTE - Instituto Universitário de Lisboa, 1649-026, Lisbon, Portugal
}

\begin{abstract}
When modeling laser wakefield acceleration (LWFA) using the particle-in-cell (PIC) algorithm in a Lorentz boosted frame, the plasma is drifting relativistically at $\beta_{b} c$ towards the laser, which can lead to a computational speedup of $\sim \gamma_{b}^{2}=\left(1-\beta_{b}^{2}\right)^{-1}$. Meanwhile, when LWFA is modeled in the quasi-3D geometry in which the electromagnetic fields and current are decomposed into a limited number of azimuthal harmonics, speedups are achieved by modeling three dimensional (3D) problems with the computational loads on the order of two dimensional $r-z$ simulations. Here, we describe a method to combine the speed ups from the Lorentz boosted frame and quasi-3D algorithms. The key to the combination is the use of a hybrid Yee-FFT solver in the quasi3D geometry that significantly mitigates the Numerical Cerenkov Instability (NCI) which inevitably arises in a Lorentz boosted frame due to the unphysical coupling of Langmuir modes and EM modes of the relativistically drifting
\end{abstract}

Email address: tpc02@ucla.edu (Peicheng Yu)

Preprint submitted to J. Comp. Phys.

April 6, 2016

(C) 2016. This manuscript version is made available under the Elsevier user license http://www.elsevier.com/open-access/userlicense/1.0/ 
plasma in these simulations. In addition, based on the space-time distribution of the LWFA data in the lab and boosted frame, we propose to use a moving window to follow the drifting plasma, instead of following the laser driver as in the LWFA lab frame simulation, in order to further reduce the computational loads. We describe the details of how the NCI is mitigated for the quasi-3D geometry, the setups for simulations which combine the Lorentz boosted frame, quasi-3D geometry, and the use of a moving window, and compare the results from these simulations against their corresponding lab frame cases. Good agreement is obtained among these sample simulations, particularly when there is no self-trapping, which demonstrates it is possible to combine the Lorentz boosted frame and the quasi-3D algorithms when modeling LWFA. We also discusse the preliminary speedups achieved in these sample simulations.

Keywords: PIC simulation, hybrid Maxwell solver, relativistic plasma drift, numerical Cerenkov instability, quasi-3D algorithm, Lorentz boosted frame, moving window

\section{Introduction}

Laser wakefield acceleration (LWFA) [1] offers the potential to construct compact accelerators that have numerous potential applications, including the building blocks for a next generation linear collider and the electron beam source for ultra-compact XFELs. It has thus attracted extensive interest, and the last decade has seen an explosion of experimental results. Fully nonlinear particle-in-cell (PIC) simulations have been instrumental in this progress as an aid in designing new experiments, in interpreting experimental results, and in testing new ideas. Furthermore, developing predictive theoretical models is challenging due to the strong nonlinear effects that are present in the blowout regime of LWFA [2]; therefore numerical simulations are also critical in exploring the physics of LWFA. Particle-in-cell simulations have been extensively applied in LWFA research because the PIC algorithm follows the self-consistent interactions of particles through the electromagnetic (EM) fields directly calculated from the full set of Maxwell equations. When modeling LWFA using the PIC algorithm the laser wavelength needs to be resolved which is usually on the scale of $1 \mu \mathrm{m}$; meanwhile, the length of the plasma column that the laser propagates through can be on the scale of $10^{4}$ to $10^{6} \mu \mathrm{m}$. As a result of this disparity in cell size and propagation 
distance, full three-dimensional (3D) PIC simulations of LWFA can be very CPU-time consuming. To capture the key physics while reducing the computation time, reduced models are continually being proposed. These include models that combine the ponderomotive guiding center with full PIC for the wake [3] or with the quasi-static approach $[4,5]$. However, these models cannot as yet model full pump depletion lengths, and the quasi-static approach cannot model self-injection.

Recently, two methods have been proposed that can speed up the LWFA simulation without losing key physics in the modeling of LWFA. One method is the Lorentz boosted frame technique [6]. In this method the LWFA simulations are performed in an optimized Lorentz boosted frame with velocity $v_{b}$, in which the length of the plasma column is Lorentz contracted, while the laser wavelength is Lorentz expanded. Assuming the reflection of the laser light is not important in the lab frame, then in a properly chosen Lorentz transformed frame the time and space scales to be resolved in a numerical simulation are minimized, and savings of factors that scales as $\gamma_{b}^{2}=\left(1-v_{b}^{2} / c^{2}\right)^{-1}$ can be achieved.

Another method that has been recently proposed is to decompose the EM fields and current density into a Fourier series in the azimuthal angle $\phi$,

$$
\begin{aligned}
\vec{F}(r, z, \phi)= & \operatorname{Re}\left\{\sum_{m=0} \vec{F}^{m}(r, z) e^{i m \phi}\right\} \\
= & \vec{F}^{0}(r, z)+\operatorname{Re}\left\{\vec{F}^{1}\right\} \cos \phi-\operatorname{Im}\left\{\vec{F}^{1}\right\} \sin \phi \\
= & \operatorname{Re}\left\{\vec{F}^{2}\right\} \cos (2 \phi)-\operatorname{Im}\left\{\vec{F}^{2}\right\} \sin (2 \phi) \\
& +\ldots
\end{aligned}
$$

and truncate the expansion at a low $m$ value [7]. This expansion is substituted into Maxwell's equations to generate a series of equations for the complex amplitudes for each harmonic. The harmonics are then summed to get the total fields. The particles are pushed in 3D Cartesian geometry and are then used to obtain the complex amplitudes for each harmonic of the current. This method can reduce the computational costs of modeling 3D problems with low azimuthal asymmetry to that on the order of $2 \mathrm{D} r-z$ simulations.

It was pointed out in Ref. $[9,10]$ that it would be intriguing to combine these two methods in order to combine the speedups provided by each. Similar to full PIC simulations in the Cartesian geometry, it was found that 
in the quasi-3D geometry one of the main obstacles to performing Lorentz boosted frame simulations is the multi-dimensional Numerical Cerenkov Instability (NCI) $[11,12,13,14]$ that inevitably arises due to the unphysical coupling between Langmuir modes (main and aliasing) and EM modes of the relativistic drifting plasma in the simulations. The coupling arises in the Lorentz boosted frame between modes which are purely longitudinal (Langmuir modes) and purely transverse (EM modes) in the lab frame. The coupling occurs at specific resonances $(\omega-2 \pi \mu / \Delta t)=\left(k_{z}-2 \pi \nu_{z} / \Delta z\right) v_{b}$ where $\mu$ and $\nu_{z}$ are the time and space aliases and $\Delta t$ and $\Delta z$ are the time step and grid size respectively, and $\omega$ and $k_{z}$ are the frequency and wave number in $\hat{z}$ direction .

While the multi-dimensional NCI theory in Cartesian coordinates has been well studied (see e.g. $[12,13,14,15,16,17,18]$ ), there are currently no analytical expressions for the numerical dispersion relation of a relativistic plasma drift in the quasi-3D geometry. However, OSIRIS [22] simulations have shown that its behavior in the quasi-3D $r-z$ geometry is very similar to that in the Cartesian geometry. It was therefore recently proposed and demonstrated that a hybrid Yee-FFT solver could be used to suppress the NCI in the Cartesian and quasi-3D geometries [18]. In the regular Yee (a finite difference) solver in a quasi-3D geometry [7, 21], Maxwell equations are solved in $(r, z)$ space for each azimuthal mode $m$. In the hybrid Yee-FFT solver, we perform a (discrete) Fourier transform in the drifting direction of the plasma (denote as $\hat{z}$ direction), and solve Maxwell equations in $k_{z}$ space for each azimuthal mode $m$; meanwhile, in the $\hat{r}$ direction the derivatives are represented as second order finite difference operators on a Yee grid. The current is corrected to maintain the correctness of Gauss' Law. When Maxwell's equations are solved in this way, the corresponding NCI modes can be systematically eliminated by applying similar strategies used for a multidimensional spectral Maxwell solver [14, 17]. The fastest growing modes of the NCI at $\left(\mu, \nu_{1}\right)=(0, \pm 1)$ can be conveniently suppressed by applying a low-pass filter in the current, the highly localized $\left(\mu, \nu_{z}\right)=(0,0)$ NCI modes can be moved away from physical modes by reducing the time step, and can be subsequently eliminated by modifying the EM dispersion at the $k_{z}$ range where the $\left(\mu, \nu_{z}\right)=(0,0)$ NCI modes are located. Furthermore, higher order spatial aliasing NCI modes can be mitigated by applying higher order particle shapes. In this paper, we present OSIRIS simulation results which show that Lorentz boosted simulations of LWFA can be performed in this geometry with no evidence of NCI. It is worth noting that recently a PIC algorithm 
based on a fully spectral solver in quasi-3D geometry has been proposed by Lehe et. al. [23] and was demonstrated with a single-node algorithm.

In addition, according to how the lab frame information is located in the $\left(z^{\prime}, t^{\prime}\right)$ space, we show that the computational loads can be further reduced by applying a moving window in the boosted frame simulation. In the boosted frame the window follows the plasma as opposed to the laser, which is the case when using a moving window in the lab frame.

The remainder of this paper is organized as follows: in section 2.1 we briefly discuss the hybrid Yee-FFT solver in quasi-3D geometry, and the corresponding NCI mitigation strategies. In section 3, we discuss the simulation setups for modeling LWFA in the Lorentz boosted frame. We discuss the distribution of the data needed for the reconstruction of lab frame information with an emphasis on showing that using a moving window in the direction of the plasma drift can further reduce the computational load. We then show sample quasi-3D simulations of LWFA in the Lorentz boosted frame in section 4, and compare the results with the corresponding 3D boosted frame and lab frame data. In particular, we concentrate on the study of the laser driver evolution as it propagates through the plasma. Good agreement is obtained when comparing the driver evolution in lab frame against that obtained from the boosted frame simulation. This demonstrates the feasibility of combining Lorentz boosted frame technique, quasi-3D algorithm, and a moving window. We also discuss the preliminary speedups achieved in these sample simulations. The results are summarized in section 5 .

\section{NCI elimination scheme in quasi-3D geometry}

\subsection{Quasi-3D hybrid Yee-FFT solver}

A key issue that needs to be addressed when performing LWFA simulations in a Lorentz boosted frame is the existence of a violent numerical instability, called the Numerical Cerenkov Instability (NCI). The NCI arises when a plasma drifts relativistically on the grid. There has been much recent progress in identifying the NCI as the source of the instability, in deriving the numerical dispersion relations and determining growth rates, and in identifying mitigation strategies $[11,12,13,14,15,16,17,18]$. In Ref. [18] a hybrid Yee-FFT solver was proposed for the elimination of the NCI in the Cartesian geometry. In this solver, Maxwell equations are Fourier transformed in the drifting direction of the plasma (denoted as the $\hat{z}$ direction). The fields are solved in the corresponding $\left(k_{z}, x, y\right)$ space, where conventional second 
order finite difference operators on a Yee mesh are used in $(x, y)$. When Maxwell equations are solved in this way, the corresponding EM dispersion of the solver leads to NCI patterns that are very similar to those from a fully spectral Maxwell solver in which Maxwell equations are solved in multidimensional $\vec{k}$-space. Therefore one can systematically eliminate the main and first spatial aliasing NCI using approximately the same strategies developed for a fully spectral solver. More importantly, the hybrid Yee-FFT solver works for both Cartesian geometry $(z, x, y)$, and quasi-3D geometry $(z, r, \phi)$. Although at present there is no rigorous theory on the NCI in the quasi-3D geometry, it has been found through OSIRIS simulations that the NCI patterns and growth rates are very similar to its counterpart in Cartesian 2D geometry [9]. Therefore, the idea of hybrid Yee-FFT solver can be readily applied to the quasi-3D geometry.

When the Maxwell solver is modified from a standard Yee solver to a hybrid Yee-FFT solver, essentially the spatial finite difference operator in the $\hat{z}$ direction is modified from second-order accuracy (derived from its finite difference form) into a greater than $N$-th order accuracy. However, in OSIRIS (and most of the modern PIC codes) the $\vec{E}$ and $\vec{B}$ fields are advanced using Faraday's Law and Ampere's Law, while Gauss's Law is satisfied by applying a charge conserving current deposition scheme [8, 24, 25]. This scheme begins by calculating the current using the charge conserving current deposit scheme of $[24,25]$ for a purely $r-z$ code. It then uses this as a common factor in the amplitude for each azimuthal harmonic of $J_{z}$ and $J_{r}$ together with a factor that depends on the particle position in $\phi$ at the half time step; and it uses this together with the particle motion in $\phi$ to get $J_{\phi}$ for each harmonic (see section 3.2 of Ref. [8] for more details). If the continuity equation is rigorously satisfied at each time step then by taking the finite difference version of the divergence of Ampere's law, Gauss' Law is seen to be satisfied if it is satisfied at $t=0$.

However, the rigorous charge conserving current deposit is known only for second order finite difference operators in the $\hat{z}$ direction. Therefore, when we use a FFT for the differential operator along $\hat{z}$ direction in Faraday's and Ampere's Law, we need to modify the current appropriately so the continuity equation is still true for the modified differential operator. To accomplish this, for each azimuthal mode of current $\vec{J}^{m}(z, r)$ obtained from the charge conserving current deposition scheme described in [8], we Fourier transform 
it along $\hat{z}$-direction, and then apply a correction with the form,

$$
\tilde{J}_{z}^{m}\left(k_{z}, r\right)=\frac{[k]_{z}}{k_{z}} J_{z}^{m}\left(k_{z}, r\right)
$$

where

$$
[k]_{z}=\frac{\sin k_{z} \Delta z / 2}{\Delta z / 2}
$$

is the second order first spatial finite difference operator. This correction ensures the satisfaction of Gauss' Law throughout the simulation, as will be discussed shortly afterwards.

Each azimuthal mode of the EM fields are initially stored in the memory in $(z, r)$ space, and are advanced in $\left(k_{z}, r\right)$ space. We Fourier transform $\vec{E}$ and $\vec{B}$ along $\hat{z}$-direction, and solve Faraday's Law and Ampere's Law for each azimuthal mode $m$, and each Fourier mode $k_{z}$, using the corrected current as the source term,

$$
\begin{aligned}
\partial_{t} B_{r}^{m} & =-\frac{i m}{r} E_{z}^{m}-i k_{z} E_{\phi}^{m} \\
\partial_{t} B_{\phi}^{m} & =i k_{z} E_{r}^{m}+\partial_{r} E_{z}^{m} \\
\partial_{t} B_{z}^{m} & =-\frac{1}{r} \partial_{r}\left(r E_{\phi}^{m}\right)+\frac{i m}{r} E_{r}^{m} \\
\partial_{t} E_{r}^{m} & =\frac{i m}{r} B_{z}^{m}+i k_{z} B_{\phi}^{m}-J_{r}^{m} \\
\partial_{t} E_{\phi}^{m} & =-i k_{z} B_{r}^{m}-\partial_{r} B_{z}^{m}-J_{\phi}^{m} \\
\partial_{t} E_{z}^{m} & =-\frac{1}{r} \partial_{r}\left(r B_{\phi}^{m}\right)-\frac{i m}{r} B_{r}^{m}-\tilde{J}_{z}^{m}
\end{aligned}
$$

Here $\vec{E}^{m}, \vec{B}^{m}$, and $\vec{J}^{m}$ are all in $\left(k_{z}, r\right)$ space. Note that $\partial_{t}$ and $\partial_{r}$ adopt the conventional finite difference form as in the Yee solver. The code is gridless in $\phi$ so $\partial_{\phi}$ is replaced with $i m$. The fields are then transformed back to $(z, r)$ space, summed over $m$ modes, and gathered for the particle pushing.

The reasoning behind the current correction Eq. (1) is that the charge conserving current deposition scheme described in [8] ensures that

$$
\partial_{t} \rho^{m}\left(k_{z}, r\right)+i[k]_{z} J_{z}^{m}\left(k_{z}, r\right)+\nabla_{r} \cdot J_{r}^{m}\left(k_{z}, r\right)=0
$$


where $[k]_{z}$ is given in Eq. (2)Therefore this correction ensures that Gauss's Law in the hybrid solver

$$
i k_{z} E_{z}^{m}\left(k_{z}\right)+\nabla_{r} \cdot E_{r}^{m}(z)=4 \pi \rho^{m}\left(k_{z}, r\right)
$$

is satisfied throughout the simulation if it is satisfied at $t=0$.

\subsection{Elimination of Numerical Cerenkov instability}

We have found previously that the NCI pattern for the quasi-3D hybrid Yee-FFT solver is similar to its counterpart in the Cartesian 3D geometry [9, 18]. As a result, we can apply approximately the same mitigation strategies used for the fully spectral solver in Cartesian geometry to systematically eliminate the NCI modes for this solver [14, 17].

We first eliminate the fastest growing $\left(\mu, \nu_{z}\right)=(0, \pm 1)$ modes $\left(\nu_{z}\right.$ is the spatial aliasing in $\hat{z}$ direction) by applying a low-pass filter in the current. The filter covers the entire $k_{z}$ range in which the $\left(\mu, \nu_{z}\right)=(0, \pm 1)$ NCI modes reside to prevent an unphysical exponential energy growth in these $k_{z}$ modes. This can be efficiently accomplished since the current density is already in $k_{z}$ space after the Fourier transform. For instance, in the sample simulation in section 4.2 we are using a low pass filter that has the following expression:

$$
F\left(k_{z}\right)=\left\{\begin{array}{l}
1, \quad\left|k_{z}\right|<f_{l} k_{g z} \\
\sin ^{2}\left(\frac{k_{z}-f_{u} k_{g z}}{f_{l} k_{g z}-f_{u} k_{g z}} \frac{\pi}{2}\right), \quad f_{l} k_{g z} \leq\left|k_{z}\right| \leq f_{u} k_{g z} \\
0, \quad f_{u} k_{g z}<\left|k_{z}\right|
\end{array}\right.
$$

where $k_{g z}=2 \pi / \Delta z$. This filter cuts off all the $k_{z}$ modes larger than $f_{u} k_{g z}$, while allowing modes smaller than $f_{l} k_{g z}$ to go through the filter. A sine square function connects the two regions to ensure a smooth filtering function. The filter parameters $f_{l}$ and $f_{u}$ are listed in Table 1 and 2 .

The second fastest growing NCI modes $\left(\mu, \nu_{z}\right)=(0,0)$ can be eliminated by reducing the time step, and then slightly modifying the $k_{z}$ operator to create a small bump in the dispersion relation to precisely avoid intersections between the main EM modes and main Langmuir modes that are highly localized in $k_{z}$ [17]. When determining the simulation time step, we first choose a time step such that the $\left(\mu, \nu_{z}\right)=(0,0)$ NCI modes are significantly far away from the physical modes. The time step we used for the simulations presented in this paper are $\Delta t=\Delta z / 4$. After that, we apply the $[k]_{z}$ modification in the highly localized $\left|k_{z}\right|$ range. This modification makes the 
growth rate of the $\left(\mu, \nu_{z}\right)=(0,0)$ NCI modes to be zero in theory. The $[k]_{z}$ modification is straightforward in a hybrid Yee-FFT solver since we are essentially solving the Maxwell equation in $k_{z}$ space. In the sample simulation presented in section 4.2 we applied the following correction to the $[k]_{z}$ operator

$$
[k]_{z}=\left\{\begin{array}{l}
k_{z}+\Delta k_{\max } \cos ^{2}\left(\frac{k z-k_{z m}}{k_{z l}-k_{z m}} \frac{\pi}{2}\right), \quad k_{z l}<\left|k_{z}\right|<k_{z u} \\
k_{z}, \quad \text { otherwise }
\end{array}\right.
$$

where $k_{z u}=2 k_{z m}-k_{z l}$, and $k_{z m}, k_{z l}, \Delta k_{\max }$ are listed in Table 1 and 2 .

As for higher order NCI modes, their growth rates can be reduced if needed by applying higher order particle shapes. However, for the parameter space explored in this paper, the higher order NCI modes have growth rates several orders of magnitude smaller than the fastest growing modes, and are not seen in the simulations even when the modes with higher growth rates are suppressed. Therefore, for the simulations presented here we used the quadratic particle shapes.

Applying the strategies described above, we can systematically mitigate the NCI modes in the quasi-3D geometry. Note the Fourier transform of the current into $k_{z}$ space is not only important for the efficient filtering of the NCI modes, but also required to accurately correct (compensate) the current in $k_{z}$ space to exactly match the modified Maxwell solver. It is worth noting that it is now a common practice to modify either the Maxwell solver or the field interpolation to change the EM dispersion relation in order to obtain a more desirable dispersion relation $[12,13,14,15,16,17,18,19]$. Within these schemes, Gauss' Law is satisfied by either directly solving it (as is the case in UPIC [26, 27, 28]), or by using a current that satisfies the continuity equation through a correction (compensation) to match the current deposition scheme with the Maxwell solver (as is the case in here and in [18]).

\section{Simulation setups in the boosted frame}

The setup of a quasi-3D LWFA simulation in a Lorentz boosted frame is almost identical to its counterpart in Cartesian 2D/3D geometry. In a boosted frame with Lorentz factor $\gamma_{b}$ that moves in the propagation direction of the laser, the laser pulse is colliding with a counter-propagating relativistically drifting plasma $[29,30,31]$. Due to the Lorentz transform, the plasma density increases by $\gamma_{b}$ while the total plasma column length contracts by 
$\gamma_{b}$. The laser wavelength and pulse length stretch by $\gamma_{b}\left(1+\beta_{b}\right)$, while its Rayleigh length contracts by $\gamma_{b}$. To avoid initializing a laser with very wide transverse size due to the contracted Rayleigh length and stretched pulse length, a moving antenna is placed at the edge of the plasma boundary to inject a laser pulse into the plasma $[32,33]$.

\subsection{Relationship between lab and boosted frame data}

In LWFA simulations in the lab frame (i.e., a stationary plasma) the use of a moving window [34], which only follows the physical domain near the laser, significantly reduces the computational load. The moving window essentially drops plasma sufficiently far behind the laser and adds fresh plasma in front of the laser. This is illustrated in Fig. 1 (a) where we plot the range of space time data from a lab frame simulation. The solid box shows the total space time area while the dashed box shows the reduced area through the use of a moving window. The moving window has a length $800\left[k_{0}^{-1}\right]$, and the simulation duration is $t_{\max }=100000\left[\omega_{0}^{-1}\right]$ (where $\omega_{0}$ and $k_{0}$ are the frequency and wave number of the laser in the lab frame). We also show the simulation data that is dumped as colored lines. The data is dumped every $20000\left[\omega_{0}^{-1}\right]$. The red ends of the data lines indicate the starting end of the moving window, while the blue ends indicate the rear end. Connecting the red ends of the data lines, we obtain the $z-t$ relation for the head of the moving windows, $t=z$ (the speed of light $c$ is normalized to 1 ). The data obtained in the lab frame (assuming the code dumps data at a constant time interval) rotates in space-time in the boosted frame since the Lorentz transform is essentially a hyperbolic rotation of coordinates in Minkowski space $[31,35]$. Therefore lines of data in $\hat{z}$ taken at fixed time from a Lorentz boosted frame are rotated by the Lorentz transform, i.e, $t^{\prime}=t / \gamma_{b}-\beta_{b} z^{\prime}$. The slope of each data line now becomes $-\beta_{b}$, where $\beta_{b}=\left(1-\gamma_{b}^{-2}\right)^{-1 / 2}$ and each data line in the lab frame which belongs to the same point in time in lab frame is now spread over a range of $t^{\prime}$ and $z^{\prime}$. Interestingly, when we connect the red end of each data line in the boosted frame it still has a slope of $c$, i.e. $t^{\prime}=z^{\prime}$. The range of data in the boosted frame is shown in Figs. $1(\mathrm{~b})$, (c) and (d). The data in Fig. 1 (b), (d) corresponds to $\gamma_{b}=20$ while that in Fig. 1 (c) corresponds to $\gamma_{b}=5$. In Figs. 1 (b) and (c) we also show the smallest area (domain enclosed by dashed lines) in $t^{\prime}, z^{\prime}$ space that includes the area needed to reconstruct the lab frame data for the two different values of $\gamma_{b}$. This illustrates that the space-time area in the boosted frame can be minimized by using a moving window in this frame. In Fig. 1 (b) it is 
seen that this window moves to the left (backwards); while in Fig. 1 (c) the window moves to the right (forwards). We use such a moving window in the boosted frame OSIRIS simulations. Currently, in UPIC-EMMA boosted frame simulations in Cartesian 2D/3D geometry a stationary window is used [see Fig. $1(\mathrm{~d})][20]$.

(a)
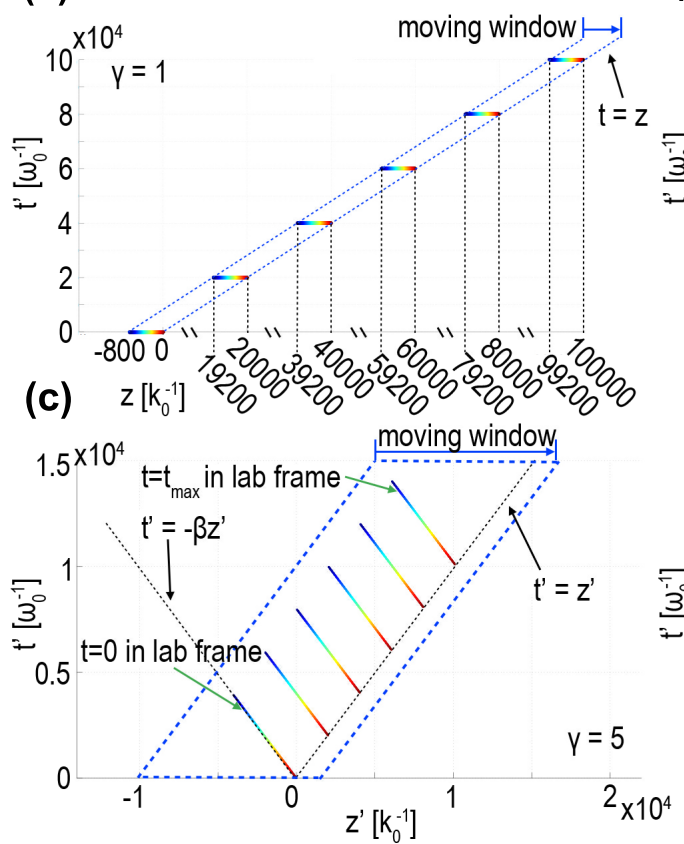

(b) $\times 10^{4}$ moving window $\quad y=20$
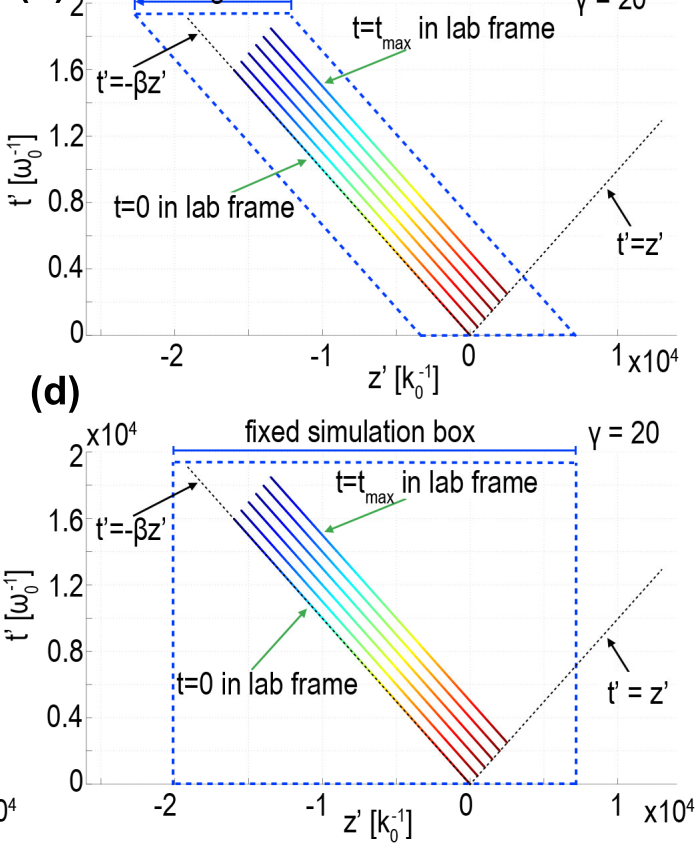

Figure 1: Range of important data in lab and boosted frame simulations. (a) Range of data in lab frame (stationary plasma) simulation with a moving window, (b) range of data in a boosted frame simulation at $\gamma_{b}=20$ with a moving window following the drifting plasma, (c) range of data in a boosted frame simulation at $\gamma_{b}=5$ with a moving window following the laser driver, and (d) range of data in a boosted frame simulation at $\gamma_{b}=20$ without a moving window.

From Fig. 1 it is evident that in lab frame simulations we usually dump data sparsely in time (large time intervals between time outputs), but the data at each grid is dumped at each time output. On the other hand, in order to recover the equivalent lab frame data in a boosted frame simulation, we need to sample boosted frame data at a much higher rate in time, but only need a small number of spatial locations. This can be seen by plotting a line across $z^{\prime}$ for a fixed $t^{\prime}$. This line only intersects the equivalent lab frame data 
at the same number of spatial locations as the number of time outputs. We typically dump the boosted frame data in a standard form (all grid points at small number of time steps) as well as the data needed to transform the results back to the lab frame (a small number of interpolated grid points at a large number of time steps). We then post-process the later data by performing the inverse rotation back into lab frame for comparison with the lab frame data. When running in the lab frame we also plot the necessary data needed to reconstruct the data into a boosted frame. This inverse construction method is useful during the development of a boosted frame code, as one can transform the lab frame data that has been extensively cross checked with theory, to the boosted frame, and compare the results against the results obtained by the boosted frame code.

\subsection{Basic setup}

In Fig. 2, we present a typical setup for a boosted frame simulation. The moving window moves from right to left following the drifting plasma. The moving antenna is also moving from right to left and injects the laser pulse from the left plasma boundary into the plasma. We place a damping section at the rear (right) end of the moving window (there is a gap between the plasma and the damping region) to damp the EM field to zero in this region. This is done because periodic boundary conditions are applied in the $\hat{z}$ direction when using the hybrid Yee-FFT solver, which requires that the EM fields need to be zero at the rear end of the simulation window to match the fields at the opposite side; otherwise the EM field at the rear end will reappear at the starting end. We note that there will be a low level of EM reflection from the damping section. In the hybrid Yee-FFT solver, the group velocity of light propagating in $\hat{z}$ direction in vacuum is greater than the speed of light, however, the drifting plasma is drifting ultra-relativistically in the same direction, the reflected wave does not have enough time to catch up with the drifting plasma for the cases of interest. Hence the physics inside the plasma will not be affected by the reflecting EM waves. We have compared cases with the moving window plus the damping regions against cases without the moving window to confirm that the moving window plus damping region works [10]. We also note that for high $\gamma_{b}$ boosted frame simulations, we find that the modified pusher described in Ref. [36] is required in order to get the evolution of the bubble correct. As pointed out in Ref. [36] the usual leap frog staggering leads to issues for the Lorentz force when there is near

cancellation of the electric and magnetic forces for relativistically moving 
particles. Determining at what $\gamma_{b}$ the modified pusher in [36] is needed is an area of future work.

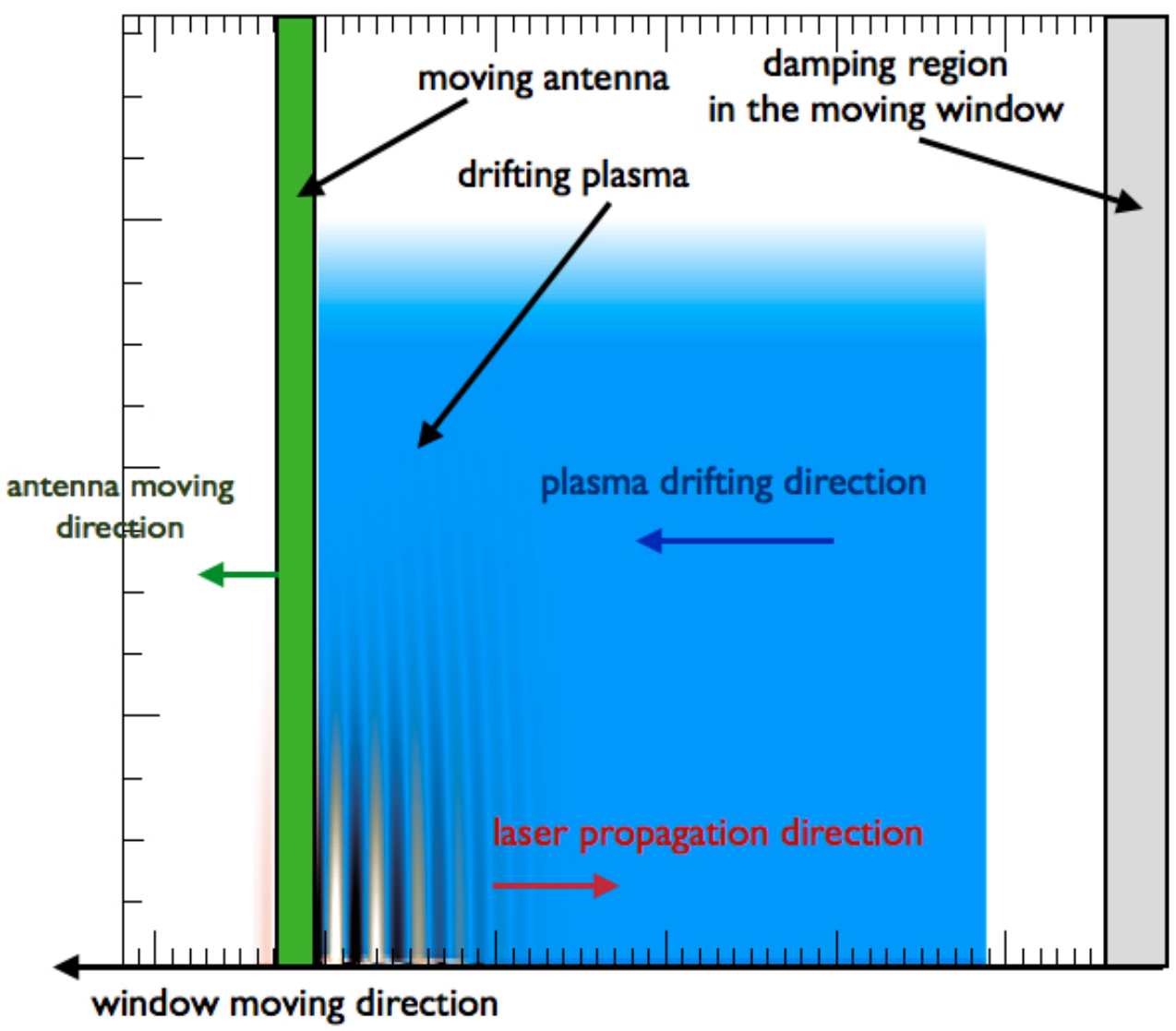

Figure 2: Simulation setup for a typical LWFA simulation in the boosted frame. The moving window follows the drifting plasma moving from right to left. A moving antenna injects laser pulse that propagates from left to right, and a damping region is located at the rear end of the moving window.

\section{Sample simulations}

In this section, we present two sets of sample simulations. We begin by comparing results from two boosted frame simulations where in one case we use full 3D OSIRIS and in the second case we use quasi-3D OSIRIS, in 
order to justify the truncation of higher azimuthal modes $m$ in the quasi3D boosted frame simulations. In both cases hybrid Yee-FFT solvers and the corresponding NCI mitigation schemes are used. The parameters match those in Ref. [2] whereby a $200 \mathrm{TW}$ laser is focused to a spot size of $19.5 \mu \mathrm{m}$ at the entrance of a $1.5 \times 10^{18} \mathrm{~cm}^{-3}$ density plasma. The FWHM pulse length of the laser was 35 fs and the normalized vector was $a_{0}=4.0$ for a linearly polarized laser or $a_{0}=4.0 / \sqrt{2}$ for a circularly polarized laser. This corresponds to a $1.3 \mathrm{GeV}$ output electron energy according to the scaling laws in Ref. [2]. The numerical parameters are shown in Table 1. We then compare the output in the boosted frame for various azimuthal mode numbers. This comparison requires the use of a post-processing algorithm which decomposes the full 3D data into azimuthal modes [39].

We then compare the data of a LWFA boosted frame simulation in quasi$3 \mathrm{D}$ lab with the corresponding quasi-3D boosted frame simulation. For these simulations we explore parameters for which a full 3D lab frame simulation is not feasible due to the large CPU hours required. The parameters correspond to a $1.8 \mathrm{PW}$ laser focused to a spot size of $45 \mu \mathrm{m}$ at the entrance of a $2.5 \times 10^{17} \mathrm{~cm}^{-3}$ density plasma. The FWHM pulse length of the laser was 130 fs and the normalized vector was $a_{0}=4.44$ for a linearly polarized laser or $a_{0}=4.44 / \sqrt{2}$ for a circularly polarized laser. This corresponds to a 10.4 $\mathrm{GeV}$ output electron energy according to the scaling laws in Ref. [2]. The numerical parameters are shown in Table 2. The data from the boosted frame simulation is transformed back to the lab frame and it is compared against the data from the lab frame simulation.

\subsection{Comparison of 3D v.s. quasi-3D boosted frame data with a $1.3 \mathrm{GeV}$ case}

When modeling LWFA in quasi-3D geometry, whether it is in the lab frame or boosted frame, the accelerating $\left(E_{z}\right)$ and focusing fields $\left(E_{r}\right.$ and $\left.B_{\phi}\right)$ in the bubble are mainly in the $m=0$ modes of the EM fields. On the other hand, the fields associated with the laser are associated with the $|m|=1$ mode of fields. Therefore, by keeping at least the $|m| \leq 1$ modes the selfconsistent evolution of the laser and wake fields can be examined when there is nearly azimuthal symmetry. For this comparison we truncate the azimuthal harmonics keeping only the $|m| \leq 1$ modes [7, 8]. More modes can be kept in principle to study laser hosing and asymmetric spot size effects as well as to test the convergence of the results. In addition, the results and the needed truncation can be verified by comparing LWFA boosted frame simulation results from the full 3D and quasi-3D geometries. To verify the azimuthal 


\begin{tabular}{lr}
\hline \hline Plasma & \\
density $n_{p}$ & $8.62 \times 10^{-4} n_{0} \gamma_{b}$ \\
length $L$ & $8.0 \times 10^{4} k_{0}^{-1} / \gamma_{b}$ \\
Laser & $86.9 k_{0}^{-1} \gamma_{b}\left(1+\beta_{b}\right)$ \\
pulse length $\tau$ & $153.0 k_{0}^{-1}$ \\
pulse waist $W$ & circular \\
polarization & 4.0 \\
normalized vector potential $a_{0}$ & \\
Quasi-3D boosted frame simulation $\left(\gamma_{b}=15.0\right)$ & $0.1 k_{0}^{-1} \gamma_{b}\left(1+\beta_{b}\right)$ \\
grid size $\Delta z=\Delta r$ & 0.125 \\
time step $\Delta t / \Delta x_{z}$ & $2048 \times 256$ \\
number of grid $($ moving window $)$ & quadratic \\
particle shape & $(2,2,16)$ \\
particle per cell $(\hat{z}, \hat{r}, \hat{\phi})$ & $(0.3,0.35)$ \\
{$[k]_{z}$ modification $\left([k]_{z l}, k_{z m}, \Delta k_{\max }\right) / k_{g z}$} & \\
low pass filter $\left(f_{l}, f_{u}\right)$ & $0.141,0.240,0.007)$ \\
Full 3D boosted frame simulation $\left(\gamma_{b}=15.0\right)$ & $0.1 k_{0}^{-1} \gamma_{b}\left(1+\beta_{b}\right)$ \\
grid size $\Delta z=\Delta r=\Delta y$ & 0.125 \\
time step $\Delta t / \Delta z$ & $2048 \times 512 \times 512$ \\
number of grid $(\operatorname{moving}$ window $)$ & quadratic \\
particle shape & $(2,2,2)$ \\
particle per cell $(\hat{z}, \hat{x}, \hat{y})$ & $(0.3,0.35)$ \\
\hline$[k]_{z}$ modification $\left([k]_{z l}, k_{z m}, \Delta k_{\max }\right) / k_{g z}$ & $(0.141,0.240,0.007)$ \\
low pass filter $\left(f_{l}, f_{u}\right)$ & \\
\hline \hline
\end{tabular}

Table 1: Parameters for the 3D and quasi-3D LWFA simulations in the Lorentz boosted frame (discussed in section 4.1). The laser frequency $\omega_{0}$ and number $k_{0}$ in the lab frame are used to normalize simulation parameters. The density is normalized to the critical density in the lab frame, $n_{0}=m_{e} \omega_{0}^{2} /\left(4 \pi e^{2}\right)$. The normalized vector potential $a_{0}$ for the laser corresponds to linear polarization. 
mode truncation, we decompose the data from the full 3D OSIRIS simulation into azimuthal harmonics and compare it against the corresponding quasi3D simulation using the parameters listed in Table 1. In Fig. 3, we plot the azimuthal decomposition of the 3D data for $E_{z}$ and $E_{r}$ at $t^{\prime}=4494.99\left[\omega_{0}^{-1}\right]$, and compare it against the corresponding quasi-3D data at the same time. For the $|m| \leq 1$ modes, very good agreement is observed. In addition, we plot the higher order $m=2,3$ modes from the 3D data in Fig. 4. We can see that the higher order modes are at least one order of magnitude smaller than those of the $m=0,1$ modes, which verifies the truncation of azimuthal harmonics at $|m| \leq 1$ in the quasi-3D simulations when the laser is nearly symmetric.

The main purpose of this section is to justify the truncation of higher azimuthal modes in quasi-3D boosted frame simulations. However, in Appendix A we show comparison of the final self-injected electron spectra in the lab frame transformed from the two boosted frame simulations, as well as from a 3D lab frame simulation [2]. Reasonable agreement is obtained but this is an area for future work.

\subsection{Comparison of quasi-3D lab frame v.s. boosted frame data with a 10.4 GeV case}

Next, we compare data from a quasi-3D LWFA simulation in the lab frame against data Lorentz transformed back to the lab frame from a quasi-3D simulation. A laser with normalized vector potential of $a_{0}=4.44$ (converted to linear polarization) with pulse length of $130 \mathrm{fs}$, and spot size of $45 \mu \mathrm{m}$ propagates into a plasma column $20.8 \mathrm{~cm}$ long (in the lab frame). We use a boosted frame with $\gamma_{b}=26.88$, and use a moving window as described earlier that follows the relativistically drifting plasma. A moving antenna injects the laser pulse into the plasma, and a damping region absorbs the EM field at the rear end of the moving window. In the upper $\hat{r}$ boundary of the simulation box we applied the Perfectly-Matched-Layer boundary condition (see Ref. [10] for more details). The plasma density is uniform along the $\hat{z}$ direction. It is uniform in $\hat{r}$ direction from $0 \leq r \leq 7000\left[k_{0}^{-1}\right]$ (where $k_{0}$ is the wave number of the laser in the lab frame), and then the density linearly ramps to zero at $r=8000\left[k_{0}^{-1}\right]$ near the $\hat{r}$ upper boundary (an additional gap of $500\left[k_{0}^{-1}\right]$ is left between the $\hat{r}$ upper plasma boundary and simulation box boundary). The linear plasma density ramp is used to prevent reflection when the laser cross the upper $\hat{r}$ plasma boundary into vacuum. Detailed simulation parameters are listed in Table 2. 

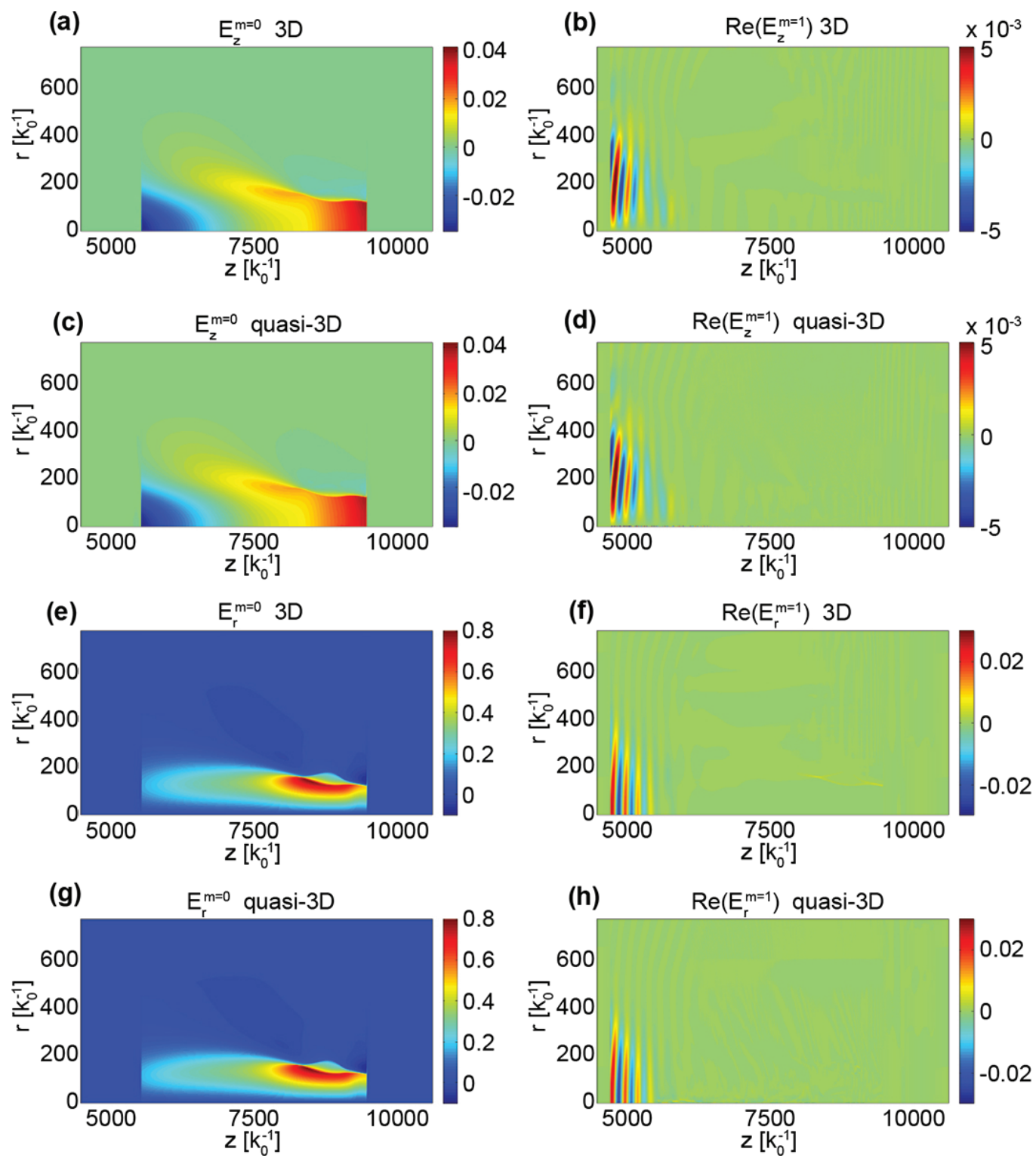

Figure 3: Comparison of simulation results in 3D and quasi-3D geometries for the $a_{0}=4.0$ (converted to linear polarization) $1.3 \mathrm{GeV}$ LWFA stage run (as discussed in section 4.1). All results are from boosted frame simulations. On the left are the $m=0$ modes of $E_{z}$ and $E_{r}$. On the right are the real part of $E_{z}^{m=1}$ and $E_{r}^{m=1}$. Results from a full 3D boosted frame case are compared against a quasi-3D OSIRIS case where only $|m| \leq 1$ modes were kept. Simulation parameters are listed in Table 1. 

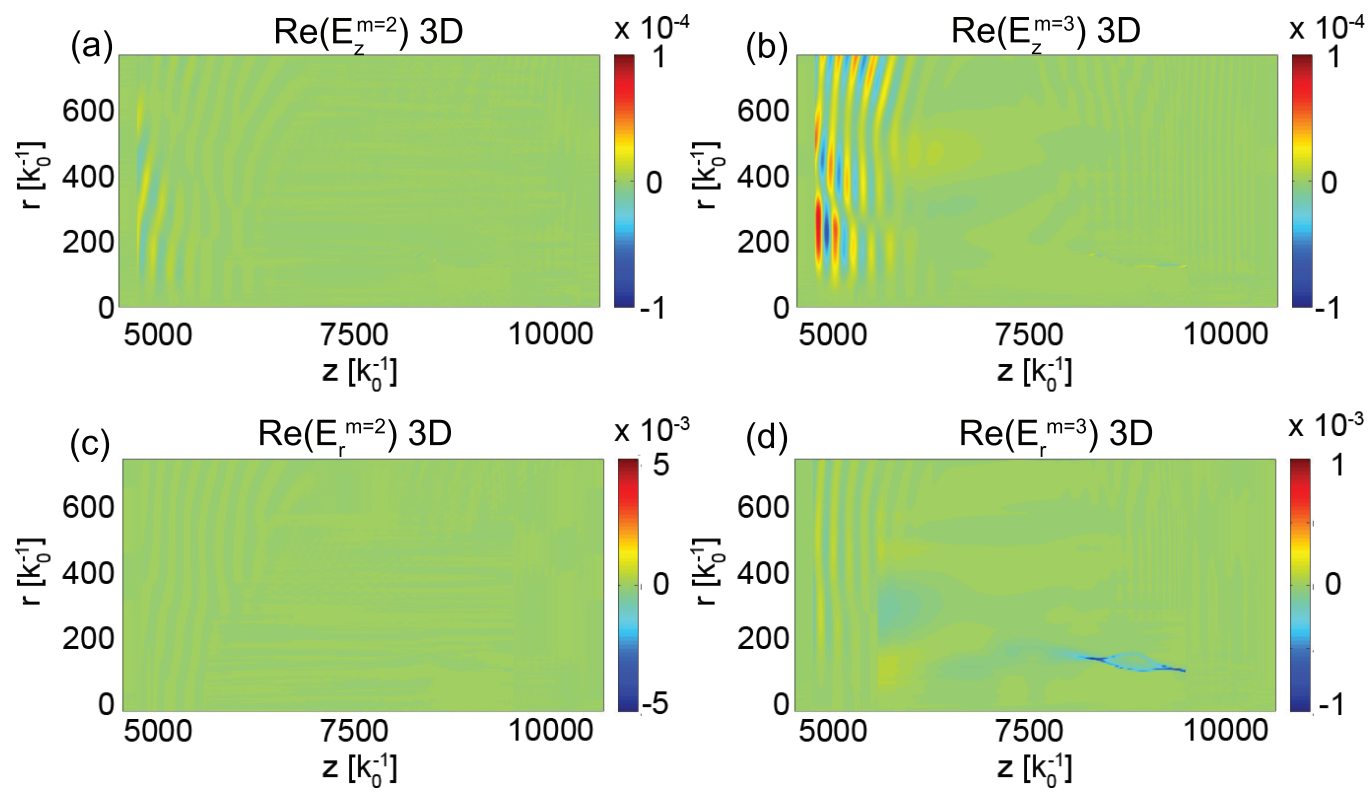

Figure 4: Higher order $m$ modes of $\operatorname{Re}\left(E_{z}\right)$ and $\operatorname{Re}\left(E_{r}\right)$ obtained from a full 3D LWFA boosted frame data (as discussed in section 4.1). On the left are $\operatorname{Re}\left(E_{z}\right)$ and $\operatorname{Re}\left(E_{r}\right)$ for mode $m=2$, while on the right are $\operatorname{Re}\left(E_{z}\right)$ and $\operatorname{Re}\left(E_{r}\right)$ for mode $m=3$. The simulation parameters used are listed in Table 1. 


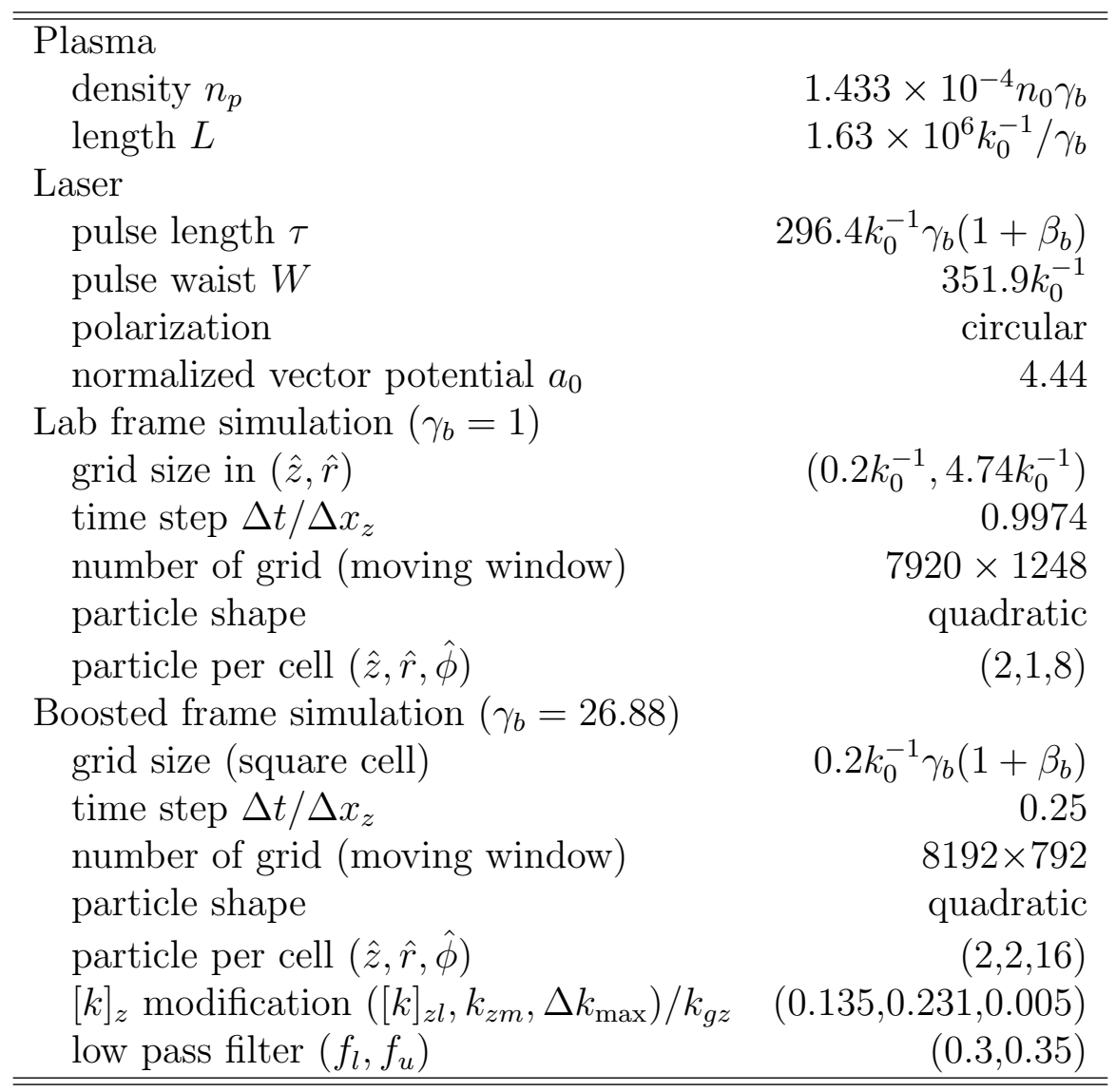

Table 2: Parameters for the quasi-3D LWFA simulations in the lab frame and Lorentz boosted frame (discussed in section 4.2). The laser frequency $\omega_{0}$ and number $k_{0}$ in the lab frame are used to normalize simulation parameters. The density is normalized to the critical density in the lab frame, $n_{0}=m_{e} \omega_{0}^{2} /\left(4 \pi e^{2}\right)$. The normalized vector potential $a_{0}$ for the laser corresponds to linear polarization. 
As mentioned in section 3, in the boosted frame each azimuthal mode of the EM field is dumped frequently in time, and sparsely in space. The results can be transformed back to the lab frame for post-processing. In Fig. 5 (a) we present 2D envelope plots of the real part of $E_{r}^{m=1}$ fields. The upper plot in Fig 5 (a) is the boosted frame simulation results (transferred back to lab frame), while the lower plot is the lab frame data. The $2 \mathrm{D}$ envelope of $E_{z}^{m=0}$ fields for the two simulations are presented in Fig 5 (b). In Figs. 5 (c)-(h) we present the corresponding line out datas from the two simulations. As we can see from Fig. 5 the data from the two simulations agree well with each other, except for the area around the rear of the first bubble, which indicates that the two simulations give different self-injection results. On the other hand, the laser profiles from the two cases agree extremely well [Fig. 5 (e)-(h)]. In Fig. 5 (e) line outs of $E_{z}^{m=0}$ at various time steps are plotted, and they show that in the transformed boosted frame data there is stronger beam loading, which indicates that more charge is self-injected into the bubble. This is likely due to the difference in statistics between the lab frame simulation and boosted frame simulation. In the boosted frame a macro-particle represents $\sim 2 \gamma_{b}^{2}$ more charge than in the lab frame, while particles in the boosted frame are $\sim 2 \gamma_{b}$ times "fatter" since the grid size in the boosted frame is $\sim 2 \gamma_{b}$ times larger, and this could affect the self-injection process.

To confirm the differences are related to the self-injection process, we repeated the lab frame and boosted frame simulations in regimes with no self-injection, at $a_{0}=3.0$ (converted to linear polarization), while keeping the other parameters as listed in Table 2. In Fig. 6 (a) and (b) we show the line out of the wakefield $E_{z}^{m=0}$ at two different times in the lab frame, and in Figs. 6 (c) and (d) we show the corresponding amplitude envelope line outs of the laser profiles $\operatorname{Re}\left(E_{r}^{m=1}\right)$. We see from Fig. 6 that for this case where there is no self-injection in the lab frame simulation, the wake field results from the lab frame and boosted frame simulations agree very well. It is challenging to accurately modeling the self-injection process in the LWFA blowout regime. Determining the best practices for using the boosted frame technique to study self-injection at high $\gamma_{b}$ is an area for future work.

We plot the laser envelope and spot size obtained from the two cases in Figs. 5 (e)-(h). Excellent agreement can be seen for the two times presented in Fig. 5. Excellent agreement is also seen for the evolution of the spot size, and laser amplitude of the laser driver as it propagates through the plasma column. In Fig. 7 we show a detailed time history of the laser spot size and amplitude at the position of the laser where its amplitude is largest. Fig. 7 

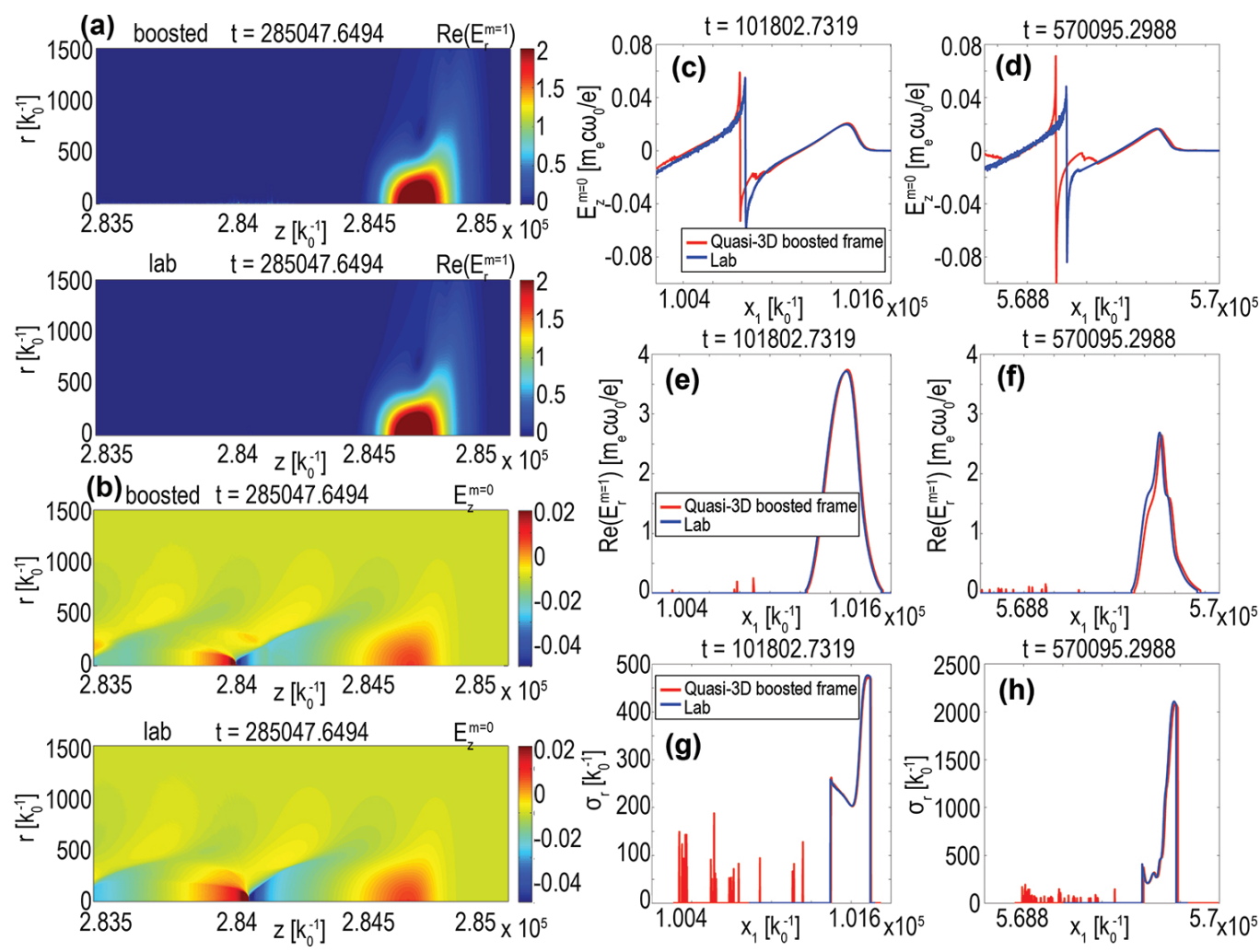

Figure 5: Simulation results for a $a_{0}=4.44$ (converted to linear polarization) $10.4 \mathrm{GeV}$ LWFA stage run (as discussed in section 4.2). (a) shows the comparison of $2 \mathrm{D}$ envelope of $\operatorname{Re}\left(E_{r}^{m=1}\right)$ field, which shows the evolution of laser driver as it propagates through the plasma; (b) shows the corresponding comparison of the amplitude of $E_{z}^{m=0}$, which shows how the wakefield of the bubble varies in the two frames due to the different self-injection results; (c), (e), and (g) are comparisons of the $E_{z}^{m=0}$ lineout, laser envelope $\operatorname{Re}\left(E_{r}^{m=1}\right)$ lineout, and laser spot size respectively at lab frame time $t=101802.7 \omega_{0}^{-1}$, while (d), (f), (h) are the corresponding plots at $t=570095.3 \omega_{0}^{-1}$. The simulation parameters used are listed in Table 2. 

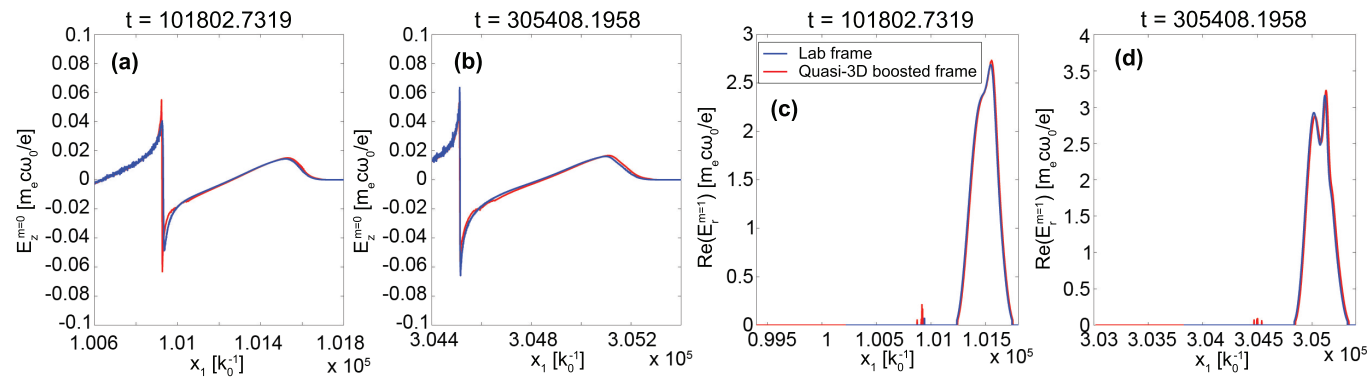

Figure 6: Line outs of wakefield $E_{z}^{m=0}$ and line outs laser field envelope of $\operatorname{Re}\left(E_{r}^{m=1}\right)$ at various lab frame time for a $a_{0}=3.0$ case (as discussed in section 4.2). Since there are no self-injection in the lab frame for this case, much better agreements are obtained for the wakefield part. The simulation parameters used are listed in Table 2.

clearly shows that very accurate results can be obtained when using Lorentz boosted frame technique in quasi-3D geometry to study the evolution of laser driver in the plasma.

We have not yet attempted to optimize choices of parameters or the algorithm itself. However, it is still useful to compare the total CPU hours for the limited set of lab and boosted frame simulations presented in the paper. The quasi-3D lab frame simulations presented in section 4.2 used around 1.6 million CPU hours. Load balancing significantly reduced the performance, and a corresponding full 3D simulation (using 8 particles per cell) would take around 300 million CPU hours in theory. Meanwhile the corresponding quasi-3D boosted frame simulation takes 2000 CPU hours. The speedup from the quasi-3D lab frame is around 800 . Note when calculating the speedup we take into account the fact that the transverse resolution, and particle per cell are different in the two simulations. Correspondingly the speedup achieved from the full 3D lab frame simulation to quasi-3D boosted frame is on the order of 100,000. Note if the full 3D simulation was run on large number of processors then load balancing issues can sometimes increase the CPU hours by a factor of between 5 and 10 . We note the theoretical speed up for boosted frame simulations is actually not straightforward to calculate as it depends on $\gamma_{b}$, the plasma length, and the laser pulse length. In addition, load imbalance is another factor that would greatly affect the speed up. 

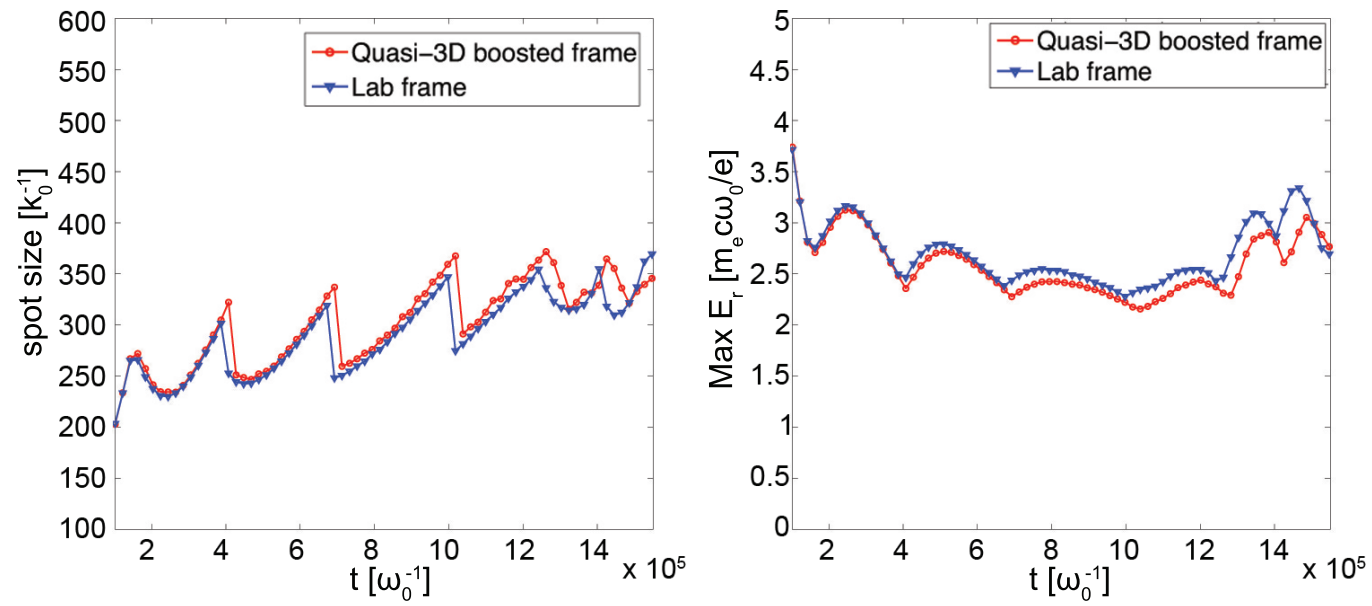

Figure 7: Evolution of the laser spot size and peak amplitude (discussed in section 4.2). (a) shows the comparison of laser spot size evolution as the laser propagates into the plasma for the two frames. The laser spot size are defined at the location where the laser has the maximum amplitude. The corresponding maximum laser amplitude evolution is shown in (b). The simulation parameters used are listed in Table 2.

\section{Summary}

In this paper, we described how it is possible to perform LWFA simulations in Lorentz boosted frames using the quasi-3D algorithm. In order to carry out high fidelity Lorentz boosted frame simulations in this geometry is the use of a hybrid Yee-FFT solver that solves the Maxwell equation in $k_{z}$ space in the direction that the plasma drifts, while keeping the second order finite-difference operators in the transverse directions as in a conventional Yee solver. Using this Maxwell solver we can then use the same strategies for eliminating main and first spatial NCI in Cartesian geometry to significantly mitigate them in the quasi-3D geometry. For the parameters that we have simulated this appears to have practically eliminated the NCI. At the same time all other features of OSIRIS are also available including single core optimization and high parallel scalability (along the non-drifting direction). Future work is planned to improve the parallel scalability in the drifting direction. A current correction is applied to ensure the code rigorously conserves charge. In addition, we analyzed the space-time area of the lab and boosted frame simulation data. We showed how using a moving window

which follows the drifting plasma in the boosted frame the further reduce 
the computational load. We were able to combine Lorentz boosted frame technique with quasi-3D algorithm, together with moving window technique, leading to significant speed up and potential unprecedented speed up for the modeling of LWFA.

We presented comparisons of lab frame against boosted frame simulation results for a $10.4 \mathrm{GeV}$ LWFA example that operates in the blowout regime. It was shown that the evolution of the laser driver in the plasma can be very well reproduced by the boosted frame simulation. We also found that the self-injection process is different in the boosted frame. This is probably due to the difference in statistics between the simulations in the two frames since in the boosted frame each macro-particle represent many more real particles then in the corresponding lab frame simulation. We found excellent agreement between the lab and boosted frame results for the wake fields when $a_{0}$ was reduced to avoid self-injection. An area of future work is to systematically explore methods to accurately model self-injection process in the Lorentz boosted frame simulation. Another area is the integration of this algorithm into our GPU and Intel-Phi enabled version of OSIRIS.

\section{Acknowledgments}

This work was supported by US DOE under grants DE-SC0008491, DESC0008316, DE-FG02-92ER40727, and by the US National Science Foundation under the grant ACI 1339893, OCI 1036224, and by NSFC 11425521, 11535006, 11175102, 11375006, and Tsinghua University Initiative Scientific

Research Program, and by the European Research Council (ERC-2010-AdG Grant 267841). Simulations were carried out on the UCLA Hoffman2 and Dawson2 Clusters, and on Hopper Cluster of the National Energy Research Scientific Computing Center, and on Blue Waters cluster at National Center for Supercomputing Applications at UIUC.

\section{Appendix A. Comparison of final spectrum of injected particles of $1.3 \mathrm{GeV}$ case}

In this Appendix, we present the comparison of the final spectrum in the lab frame of the injected particles for the $1.3 \mathrm{GeV}$ simulation in the 3D lab frame, 3D boosted frame, and quasi-3D boosted frame that are previously discussed in section 4.1. The parameters for the 3D/quasi-3D boosted frame 


\begin{tabular}{lr}
\hline \hline Plasma & \\
density $n_{p}$ & $8.62 \times 10^{-4} n_{0}$ \\
length $L$ & $8.0 \times 10^{4} k_{0}^{-1}$ \\
Laser & \\
pulse length $\tau$ & $86.9 k_{0}^{-1}$ \\
pulse waist $W$ & $153.0 k_{0}^{-1}$ \\
polarization & circular \\
$\quad$ normalized vector potential $a_{0}$ & 4.0 \\
Simulation parameters & \\
grid size $\left(\Delta x_{1}, \Delta x_{2,3}\right)$ & $\left(0.2 k_{0}^{-1}, 3.4 k_{0}^{-1}\right)$ \\
time step $\Delta t / \Delta x_{1}$ & 0.995 \\
number of grid (moving window) & $4000 \times 512 \times 512$ \\
particle shape & quadratic \\
\hline \hline
\end{tabular}

Table A.3: Parameters for the 3D lab frame LWFA simulation (discussed in section 4.1 and Appendix A). The laser frequency $\omega_{0}$ and number $k_{0}$ in the lab frame are used to normalize simulation parameters. The density is normalized to the critical density in the lab frame, $n_{0}=m_{e} \omega_{0}^{2} /\left(4 \pi e^{2}\right)$. The normalized vector potential $a_{0}$ for the laser corresponds to linear polarization.

simulations are listed in Table 1, and the corresponding lab frame simulation parameters are listed in Table A.3 below.

We can see that the final particle energy spectrum for the three cases agree relatively well. The two boosted frame simulation almost overlap each other, while the lab frame data is shifted to higher energy. We note that determining how the Lorentz boosted frame factor $\gamma_{b}$, cell sizes, time step, particle per cell, and other simulation parameters affect the modeling of LWFA in the Lorentz boosted frame remains an open question, and will be part of our future work.

\section{References}

\section{References}

[1] T. Tajima, J.M. Dawson, Phys. Rev. Lett. 43 (1979) 267

[2] W. Lu, et al., Phys. Rev. Spec. Top., Accel. Beams 10 (2007) 061301.

[3] D.F. Gordon, W.B. Mori, T.M. Antonsen, IEEE Trans. Plasma Sci. 28 (2000) 1135. 


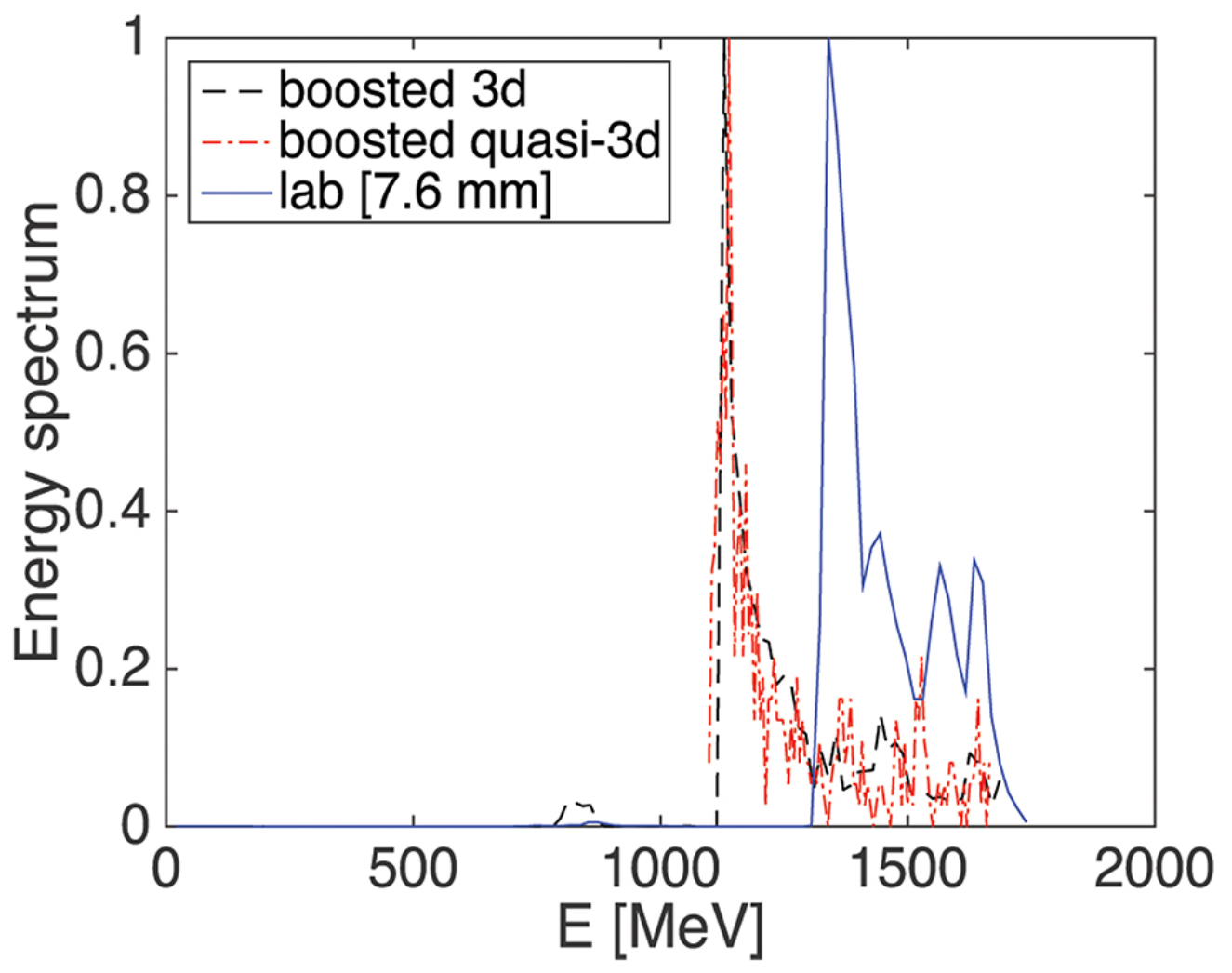

Figure A.8: Comparison of the final spectrum of the injected particles for the $1.3 \mathrm{GeV}$ simulation in the 3D lab frame, 3D boosted frame, and quasi-3D boosted frame for the $a_{0}=4.0$ (converted to linear polarization) $1.3 \mathrm{GeV}$ LWFA stage run (as discussed in section 4.1 and Appendix A). The simulation parameters used are listed in Table 1 and A.3. 
[4] P. Mora, T.M. Antonsen, Phys. Plasmas 4 (1997) 217.

[5] C. Huang, et al., J. Comp. Phys. 217 (2006) 658.

[6] J. -L. Vay, Phys. Rev. Lett. 98, 130405 (2007)

[7] A.F. Lifschitz, et al., J. Comput. Phys. 228 (2009) 1803.

[8] A. Davidson, et. al., J. Comp. Phys. 281(2015), 1063.

[9] P. Yu, et. al., in Proc. Advanced Accelerator Concept Workshop 2014, San Jose, California, USA.

[10] P. Yu, et. al., in Proc. International Particle Accelerator Conference 2015, Richmond, Virginia, USA.

[11] B. B. Godfrey, J. Comp. Phys. 15, 504 (1974)

[12] P. Yu et.al, in Proc. 15th Advanced Accelerator Concepts Workshop, Austin, TX, 2012, in AIP Conf. Proc. 1507, 416 (2012).

[13] B. B. Godfrey and J.-L. Vay, J. Comp. Phys., 248, 33-46 (2013)

[14] X. Xu, et. al., Comp. Phys. Comm., 184, 2503-2514 (2013)

[15] B.B. Godfrey, J.-L. Vay, I. Haber, J. Comput. Phys. 258 (2014) 689.

[16] B.B. Godfrey, J.-L. Vay, I. Haber, J. Comput. Phys. 267 (2014) 1.

[17] P. Yu, et. al., Comp. Phys. Comm., 192, 32 (2015).

[18] P. Yu, et. al., Comp. Phys. Comm., 197, 144 (2015).

[19] R. Lehe, et. al., Phys. Rev. ST - Accel. Beams 16, 021301 (2013).

[20] P. Yu, et. al., J. Comp. Phys. 266, 124 (2014).

[21] K. Yee, IEEE Trans. Antennas and Propagation, 14, 302 (1966)

[22] R. A. Fonseca, et al., in: P.M.A. Sloot, et al. (Eds.), ICCS, in: Lect. Notes Comput. Sci., Vol. 2331, 2002, pp. 34-351; R. Hemker, Ph.D. thesis, UCLA, 2000 (arXiv:1503.00276)

[23] R. Lehe, et. al., arXiv:1507.04790 (2015). 
[24] J. Villasenor, and O. Buneman, Comp. Phys. Comm. 69 (1992) 306.

[25] T. Esirkepov, Comp. Phys. Comm. 135 (2001), 144.

[26] J. M. Dawson, Rev. Modern Phys., Vol. 55, No. 2, 403 (1983)

[27] A. T. Lin, J. M. Dawson, H. Okuda, Phys. Fluids, 17, 1995 (1974).

[28] V.K. Decyk, Comput. Phys. Commun. 177 (2007) 95.

[29] S. F. Martins, R. A. Fonseca, W. Lu, W. B. Mori and L. O. Silva, Nat. Phys. 6, 311 (2010)

[30] J. -L. Vay, C. G. R. Geddes, E. Cormier-Michel, D. P. Grote, J. Comp. Phys. 230, 5908 (2011).

[31] S. F. Martins, R. A. Fonseca, L. O. Silva, W. Lu, W. B. Mori, Comp. Phys. Comm. 181, 869 (2010)

[32] J.-L. Vay, C.G.R. Geddes, E. Esarey, C.B. Schroeder, W.P. Leemans, Phys. Plasmas 18 (2011) 123103.

[33] J.-L. Vay, et al., in: Proc. 14th Advanced Accelerator Concepts Workshop, Annapolis, MD, in: AIP Conference Proceedings, 2010, pp. 244249.

[34] C.D. Decker, W.B. Mori, Phys. Rev. Lett. 72 (1994) 490.

[35] J.-L. Vay, C.G.R. Geddes, E. Cormier-Michel, Phys. Plasmas 18 (2011) 030701.

[36] J.-L. Vay, Phys. Plasmas 15, 056701 (2008).

[37] J. P. Boris, in Proc. 4th Conference on Numerical Simulation Plasmas, Naval Research Laboratory, Washington, D.C., 1970, pp. 3-67.

[38] J.-L. Vay, I. Haber, B. B. Godfrey, J. Comp. Phys. 243 (2013), 260.

[39] T. Dalichaouch, et. al., Bulletin of the American Physical Society 60 (2015). 